\title{
La maduración cerebral en el niño. El caso de la adquisición del concepto de muerte y su evolución ${ }^{1}$
}

\author{
Brain development in childhood. Acquisition and evolution of children's \\ understanding of death
}

\author{
Colomba Norero V.a
}

${ }^{a}$ Academia de Medicina. Instituto de Chile. Profesor Titular Pediatría. Universidad de Chile y UNAB

${ }^{1}$ Conferencia presentada en sesión de la Academia Chilena de Medicina. Instituto de Chile. 3 de mayo de 2017

\begin{abstract}
Resumen
Se presenta la relación entre la maduración cerebral en el niño y la adquisición de los cinco subcomponentes biológicos del concepto maduro de la muerte: Irreversibilidad, aplicabilidad, inevitabilidad, cese funcional y causalidad. Las teorías actuales plantean que el conocimiento (entre otros el de la muerte) se realiza en un proceso de oleadas confluentes, con avances y retrocesos y esto explica la diferencia muy importante que se puede encontrar en niños de la misma edad y buen coeficiente intelectual. Generalizando, niños menores de 4 años no tienen conciencia de la muerte. Posteriormente descubren, con dificultad, el concepto de irreversibilidad y hay mucha discusión sobre el orden de adquisición del resto de los subcomponentes- lo que está relacionado con la incorporación del pensamiento dualístico y cierto grado de conocimiento biológico. No hay acuerdo sobre la influencia cultural, socio económica y experiencias previas con la muerte de familiares. Alrededor de los 12 años se considera que se logra un concepto maduro. Se revisa la incorporación de elementos sobrenaturales en la literatura infantil clásica y actual, comprobándose que su presencia es más marcada en los cuentos para preescolares occidentales que orientales en el siglo XXI.
\end{abstract}

Palabras clave: Maduración cerebral; Conceptos biológicos de muerte; Niñez

\section{Keywords:}

Brain development; Biological deaths concepts; Children 
La maduración cerebral en el niño es un concepto muy abstracto y complejo que ha sido un tema tabú en la literatura.

Probablemente porque resulta difícil abordar una mente infantil desde la perspectiva de un adulto, persona que es otro, que dejó de ser niño y que ha olvidado o pretende haber olvidado como abordó el tema de la muerte.

Sin embargo, la idea de la muerte en los niños rodea todas las manifestaciones del saber humano: La música, la fotografía, las películas, los cuentos y novelas, la vida diaria. Pero vistas desde la lejanía de la adultez, con las interpretaciones de la imaginación y el conocimiento del adulto.

Los pediatras nos preocupamos del crecimiento y desarrollo como un pilar de nuestra especialidad. El otro gran tema es la diferenciación entre el niño y el adulto. El tema de la muerte en el niño generalmente lo analizamos solo desde las estadísticas de mortalidad infantil, sus causas y especialmente sus modificaciones con el avance de la civilización -que paradójicamente nos enfrenta en este siglo a las muertes violentas en la infancia derivadas del terrorismo y de las migraciones como causas importantes.

También nos preocupamos de los pequeños pacientes con enfermedad terminal y allí, por primera vez, pensamos en la muerte desde el punto de vista del niño y no desde el nuestro ${ }^{1}$. Y nos encontramos con sorpresas.

En la cultura occidental, especialmente en los siglos XVI a XIX, la muerte se consideraba un proceso natural que debía ser aceptado como tal. La organización familiar permitía una relación estrecha con la muerte "normal" -la de los ancianos- rodeados de sus familias $\mathrm{y}$ en respeto.

Así, los adultos se apoyaron legítimamente en conceptos religiosos y de la literatura para reforzar en los niños ideas sobrenaturales que mitigaran, según su parecer, el temor a la muerte. La existencia de un más allá, de una partida a un lugar mejor y feliz hizo pensar que con esto se disminuía el impacto.

Paralelamente, sin embargo, los seres sobrenaturales y hechos malvados, llenan las páginas de los libros infantiles. Los juicios de conducta, los castigos por lo hecho en vida se encuentran en las ideas religiosas.

Bien sabemos que fue una técnica de aprendizaje que hemos sufrido todos.

Debemos llegar al siglo XX, con la revolución industrial instalada y el fin de la Primera Guerra Mundial para que se produjera un cambio total en la valoración de las personas y los hechos. El cambio de costumbres se hizo marcado, la secularización y el énfasis en el desarrollo científico llevaron a un increíble crecimiento de información en las neurociencias, que a través de técnicas no invasivas logró conocer los procesos cerebrales en el propio ser humano.
Lentamente, el proceso de aprendizaje fue abordado. La neurofisiología y la psicología contribuyeron a penetrar en la mente del hombre y conocer su desarrollo cognitivo y con ello intentar entender el por qué, cuándo y cómo se producen los procesos de conocimiento de la muerte.

Por eso el propósito de esta revisión es señalar las evidencias actuales del abordaje del ser humano sobre la muerte desde un punto de vista biológico, cómo se llega a aceptarla para los otros y para sí mismo. El niño es el punto de partida.

Creo conveniente hacer un recuerdo sobre aspectos de la conducta social del niño que son resultado de su desarrollo cerebral y a su vez influyen en su capacidad cognitiva.

Se sabe que es capaz de reconocer caras y ademanes mucho antes de los 6 a 8 meses de edad que era lo habitualmente aceptado. Aquí empieza el proceso de imitación.

A los 2 años desarrolla el lenguaje y hasta los 3 años hace uso de sus distinciones emocionales, esto es, expresiones exageradas de desagrado o de felicidad, las pataletas que vemos en todos los niños por motivos fútiles o inexistentes.

Solo desde los 3 y especialmente cerca de los 7 años desarrolla los pilares de su individualidad. Antes no tiene conciencia de su propio yo. Es una etapa difícil en que empieza a desarrollar su conciencia subjetiva (yo mismo, ente aislado de los otros y de las cosas), inicia su memoria autobiográfica y ocupa rudimentariamente la teoría de la mente, esto es, se conecta con las otras mentes a través de las neuronas espejo ${ }^{2}$ pudiendo reconocer las verdaderas intenciones y emociones del interlocutor. Empieza a acatar normas.

Desde los 7 años es capaz de tener pensamiento dualístico. Pueden coexistir en él ideas contradictorias y ser aceptadas igualmente ${ }^{3}$.

En la adolescencia desarrolla un mecanismo de rechazo a lo establecido, se burla de su medio y de las costumbres y recién logra desarrollar juicios autónomos a los 20 años.

Con esta base vamos a ver cuáles fueron los hitos en el conocimiento de la muerte por el niño.

- 1939. Nagy y Anthony.

- 1963. Teoría del conocimiento. Jean Piaget.

- 1985. Descubrimiento de las neuronas espejo Marco Iacoboni. Teoría de la mente.

En 1941 Sylvia Anthony publica el libro "El descubrimiento de la muerte en la infancia y después", referente indiscutible sobre el tema ${ }^{4}$.

La imprenta donde lo editó en Londres fue arrasada en un bombardeo en la Segunda Guerra Mundial y el libro prácticamente se perdió. En los años 70 lanzó una segunda edición que es ampliamente apreciada hasta hoy. 
Su estudio se basa en 128 niños ingleses de 3 a 11 años. Lo genial de esta psicóloga fue que sus observaciones las hizo mayoritariamente mediante los informes escritos de los padres sobre reflexiones espontáneas de sus hijos sobre la muerte en el acontecer diario.

En pocas palabras, logró comprobar que bajo los 5 años los niños no consideran la muerte como un hecho irreversible.

La muerte de sus mascotas es mejor comprendida y sentida que la de los seres humanos -aún familiares muy cercanos.

Para los niños pequeños todos los objetos y elementos de la naturaleza con movimiento están vivos. La muerte está relacionada con la inmovilidad que se manifiesta en el hombre con un sueño profundo que puede significar una partida temporal.

Anthony relacionó el conocimiento de la muerte con la edad y el coeficiente intelectual del niño.

Piaget en los años 60 elaboró la "Teoría del desarrrollo cognitivo "que plantea que la adquisición de habilidades se hace por etapas secuenciales relacionadas con la edad, en forma de peldaños. Esta teoría se arraigó fuertemente en los sistemas educativos y de salud.

Al aplicarla a la comprensión de la muerte por el niño se apreció su estrecha relación con la adquisición de su capacidad de razonamiento ${ }^{5}$.

En síntesis, esta teoría plantea una primera etapa, llamada preoperacional (de 2 a 7 años de edad) en que se inicia el pensamiento simbólico que se relaciona con el lenguaje y la comunicación. Es el período del pensamiento mágico y del egocentrismo -el niño cree firmemente que es capaz de hacer que sucedan cosas con la sola fuerza de su deseo. No tiene una noción clara de su individualidad, no se siente separado de las otras personas y de las cosas, concepto que va adquiriendo paulatinamente.

En la etapa operacional concreta (desde los 7 a 11 años) su pensamiento se hace más lógico, reconociendo los impactos externos.

En la etapa operacional formal (desde los 12 años) empieza el pensamiento abstracto con capacidad de deducción hipotética y el niño es capaz de pensar en múltiples dimensiones.

Piaget ha sido muy criticado por falta de rigor estadístico en su trabajo y especialmente por subvalorar la inteligencia de los niños. Ignoró las diferencias individuales y no consideró las influencias culturales y del medio ambiente.

Sin embargo contribuyó a fijar los dos conceptos planteados en relación al desarrollo mental que se consideraron inamovibles durante décadas: Edad y capacidad cognitiva.

Desde los años 80 se reconoce que la capacidad cognitiva de los niños puede ser muy variable en cada edad, lo que hace difícil enclaustrarlo en una etapa de- terminada y se plantea que el conocimiento no se hace en peldaños sino que en ondas confluentes e incluso curvas en U en que la edad de 7 años es la parte más baja de la curva en muchos aspectos.

El énfasis en los siguientes años se ha colocado en entender el aprendizaje como procesos biológicos cerebrales resultado de mielinización, crecimiento neuronal, crecimiento acelerado del lóbulo frontal (entre los 6 y 10 años), conectividad e integración de los dos hemisferios (de los 4 a 10 años) presencia de neuronas espejo y fusiformes a los 19 años $)^{6}$.

Estos procesos explican el desarrollo del lenguaje, de la individualidad, de la categorización y la elaboración de juicios autónomos independientes del aprendizaje.

Así, las estructuras de la memoria y del proceso de información están condicionadas por la maduración cerebral y eso explica la gran variabilidad, las modificaciones de las ideas y las estrategias que utilizan los niños para entender y defenderse de fenómenos complejos ${ }^{7}$.

El conocimiento resulta de un proceso de retroceso y avance, una oscilación entre una variedad de estrategias para llegar a una nueva etapa.

El estudio actual se ha centrado en la comprensión biológica de la muerte y se considera que dicha comprensión está madura cuando se han adquirido cinco subcomponentes que se han identificado y ratificado en numerosos estudios.

Ellos son:

Irreversibilidad, Aplicabilidad, Inevitabilidad, Cese funcional y Causalidad.

No hay un acuerdo sobre el orden en que se van adquiriendo estos subcomponentes pero parece que la irreversibilidad es el primero en captarse y hasta hace poco tiempo la causalidad era el último... lo que comentaremos más adelante.

1. La irreversibilidad -en el sentido de que una vez que alguien o algo viviente (planta, animal) muere, no puede volver a la vida- es un concepto que ya puede estar adquirido en niños inteligentes desde los cuatro años. Lo relacionan con el cese de funciones como respiración y con la inmovilidad. Sin embargo es frecuente que se aprecien confusiones entre el movimiento propio o el inducido (mascota muerta flotando en el agua).

2. La aplicabilidad o universalidad es comprender que la muerte es algo que nos ocurrirá a todos los seres vivientes. Hasta los 6 años los niños no distinguen claramente las diferencias entre objetos y personas, atribuyendo a los objetos las cualidades de los seres vivientes. De allí la dificultad para captar este subcomponente.

3. La inevitabilidad es un subcomponente estrechamente ligado a la irreversibilidad y al cese funcio- 
nal. Todos vamos a morir, pero niños de hasta los 11 años, con buen coeficiente intelectual y sin niveles particulares de ansiedad, desarrollan ideas contradictorias y aceptan la muerte de los otros pero no la propia ${ }^{7}$. Esto es producto del pensamiento dual en que se entremezclan conceptos biológicos con pensamientos religiosos (vida en el más allá) o sobrenaturales (influencia de los programas "infantiles").

4. El subcomponente cese funcional se adquiere alrededor de los 4 a 6 años. Allí entienden que para vivir se necesita comer y respirar y logran atrapar mejor la irreversibilidad. Sin embargo, en un trabajo hecho en 2007, 31\% de los niños hasta los 11 años considera que los procesos mentales continúan después de la muerte. Este subcomponente es el más influenciado por las creencias culturales y religiosas.

Entender este subcomponente requiere de un bagaje de conocimientos biológicos que solo se puede adquirir en forma racional en la infancia tardía. Pero hay todo tipo de excepciones al respecto. Niños muy pequeños explican la muerte de los ancianos como que el corazón les dejó de funcionar. Este subcomponente puede estar influenciado por el medio social y la escolaridad de los padres.

5. Causalidad. Durante largo tiempo se consideró que era el subcomponente adquirido más tardíamente. El niño lograba comprender las causas internas de muerte (vejez, enfermedad) producida por cese funcional pero se le hacía más difícil entender las causales externas (violencia, terrorismo, cataclismos) Se podría pensar que ello se debía a estar relativamente ajeno a esos problemas ya que no había información tan inmediata y sin censura como la actual- aunque la violencia haya existido siempre. La diferencia es que ahora los sufre directamente, los ve todos los días por TV, los absorbe claramente en las publicaciones infantiles y los comenta en familia.

Creo que la visión de la muerte violenta, todos los días y en todos los planos debe haber producido niveles de ansiedad, mecanismos de defensa y aceptación de un hecho que no era comprensible a edades más tempranas.

Aunque parezca irónico decirlo, antes la muerte ajena era más suave para los niños.

Intentando resumir cuales son los elementos que llevan a la comprensión de la muerte por el niño, sin duda la edad y el proceso de maduración cerebral relacionado con ella son los factores clave; probablemente la experiencia directa o indirecta y el contexto sociocultural cumplan un rol.

Estas afirmaciones pretendieron ser corroboradas en una interesante tesis de Hopkins en 2014 (U. East Anglia, Norwich) en 92 niños ingleses de 3 a 11 años, con metodología muy rigurosa ${ }^{8}$.

\section{Concluyó:}

1) Desde el punto de vista biológico los niños entre 4 y 5 años tienen una comprensión menos sofisticada de la muerte.

2) El coeficiente intelectual está asociado positivamente con un concepto maduro de la muerte como evento biológico.

3) La irreversibilidad es el primer subcomponente en adquirirse.

4) No hay diferencias significativas según estado socioeconómico.

5) No es significativa la experiencia previa con la muerte de cercanos ni con experiencias religiosas.

Se puede criticar estos resultados por el bajo número de niños estudiados y su heterogeneidad social y religiosa.

\section{¿Cuánto saben los niños de la muerte?}

En el paciente pediátrico terminal hay un consenso que hasta los 3 a 4 años no habría conciencia de muerte.

En niños mayores, en que el tema puede ser explorado en condiciones más objetivas, hay un trabajo notable hecho en Nueva Zelandia por Gaab y cols. en un grupo importante de niños sanos y enfermos y sus cuidadores 9 .

Se dividieron los cuidadores en aquellos que habían abordado el tema de la muerte con los niños y en los que no lo hicieron.

Los niños se separaron por sexo, edad y conocimiento de los cinco subcomponentes de la muerte biológica, analizándose cómo era la concordancia de lo que creían los cuidadores sobre lo que sabían los niños del tema y el resultado de la encuesta que se les aplicó al personal.

Los resultados demostraron que prácticamente siempre los niños sabían más de lo que los cuidadores creían, a cualquier edad.

Más importante aun, después de la muerte de los chicos enfermos terminales aquellos cuidadores que habían conversado libremente con ello se sentían satisfechos porque sentían que los habían ayudado en esta etapa. Aquellos que no hablaron se sentían con una carga espiritual por no haberlo hecho.

Los niños enfermos desarrollan mecanismos de defensa muy especiales. Un ejemplo extremo de ello es el de una chica inglesa de 14 años con enfermedad terminal que ganó en el Parlamento en el año 2016, el derecho a ser criogenizada, a lo que se oponía su padre y apoyaba su madre (separados). La criogenización se realizó con el apoyo económico de su abuela materna. 
Estos son los niños y las familias de ahora.

La muerte no es -y no debe ser- un secreto para los niños sanos o enfermos.

Por último y como una licencia a mis intereses literarios, no puedo dejar de esbozar algunos datos sobre la influencia de los cuentos infantiles en el ideario de la muerte en el niño.

Parte de la literatura infantil clásica, aborda la violencia y la muerte sin angustias, como algo absolutamente normal. La convivencia de hadas, ogros y seres humanos se da en una forma muy natural, de salto en salto, siempre en tiempo presente como muy bien lo comenta Armando Roa en 1980 en su Presentación sobre Los cuentos de Charles Perrault ${ }^{10}$. Los personajes de estos cuentos (escritos en 1697) no se afligen ni en las peores circunstancias, no sufren con sus enfermedades y "la muerte apenas desempeña un papel notorio si es vivida a la manera de un presente inmediato... lo que todavía no llega, no existe; recuerda en parte aquello de Descartes, 'mientras pienso, existo', o sea, existe lo que se está experimentando, el resto desaparece". Esta falta del concepto de tiempo es un elemento muy importante.

Otras obras de literatura infantil, están cargadas de la visión de objetos inanimados llevados a la vida, el clásico ejemplo de Pinocho, o de seres etéreos dotados de vida eterna como Peter Pan. Todos ellos haciéndose eco de las creencias de la época en que fueron escritos.

Los niños actuales están bombardeados por los personajes de los cómics y animés, de una violencia extrema, en que las fronteras entre lo real y lo ilusorio están perdidas en su totalidad. La muerte por asesinato es cosa de todos los días y a todas horas, sean programas para adultos o para niños ${ }^{11}$.

En una revisión hecha el año 2014 de 40 libros de dibujo de ficción contemporánea, dirigido a preescolares asiáticos y europeos (la mayoría de Inglaterra y Corea) con mínimo material escrito y que cumplían con las premisas de excluir desastres naturales, se estudió la presencia en los relatos de los cinco subcomponentes de la muerte y además se revisaron los elementos sobrenaturales presentes ${ }^{12}$.

El $45 \%$ de los libros europeos y solo el 25\% de los asiáticos incluyen explicaciones sobrenaturales de la muerte (presencia de fantasmas, vida después de la muerte).

Se concluyó que los subcomponentes causalidad y cese de funciones en el contexto europeo parecen estar eficientemente integrados con los contenidos sobrenaturales de la muerte. Esta coexistencia, embebida en la correspondiente cultura y religión complementarían los conceptos biológicos de la muerte con las alternativas racionales lo que tiende aliviar la tristeza y la ansiedad.

En contraste, la literatura infantil asiática relacionada con la muerte se basa mayoritariamente en aspec- tos naturalísticos, lo que favorece comprender el subcomponente causalidad. Esto es también el resultado de las ideas religiosas imperantes en esas culturas.

La literatura para adultos tiene obras maestras que relacionan la conducta de los niños ante la muerte con efectos sobrenaturales escalofriantes como es "Otra vuelta de tuerca" de Henry James.

Para terminar quisiera señalar un cuento autobiográfico del Premio Nobel de Literatura 2013, la canadiense Alice Munro. Se llama "El ojo"13.

La autora cuenta sus impresiones cuando ella tenia entre 5 y 6 años de edad y Sadie, una muchacha de 16 a 17 años que ayudaba en los quehaceres de su casa y de la cual se hizo muy amiga, fallece una noche en un accidente de tránsito en el pequeño pueblo en que vivía. Alice es obligada por su madre a acompañarla al velorio.

No quería mirar a la muerta por lo que entrecerró los ojos pero enfrente al ataúd finalmente miró directamente a Sadie y vió que la cosa no era tan mala como la había imaginado. "Miró el cojín amarillo que hacía de almohada y cada vez con más atención a Sadie. Algo se movió. Lo ví, el párpado de mi lado se movió.

No es que se abriera, ni que quedara entornado. Se levantó imperceptiblemente, como para que, si hubiera alguien dentro de ella, pudiera ver a través de las pestañas. No me sobresalté ni me asusté en lo más mínimo. Esa imagen se fundió en ese mismo momento con todo lo que sabía de Sadie y también, en cierto modo, con la experiencia extraordinaria que se me ofrecía. Y no se me ocurrió llamar la atención de nadie ante lo que veía, porque no estaba destinado a ellos: era exclusivamente para mì".

La imagen de Sadie se desvaneció de su mente bastante rápido y cuando pensaba en ella nunca se cuestionaba lo que había visto y que creía destinado a ella.

"Lo creía como lo que crees, $y$ de hecho recuerdas, que tuviste dientes de leche y que ahora no están pero existieron de verdad. Hasta el día que, ya en mi adolescencia, supe con una vaga sensación de vacio en mis entrañas que había dejado de creerlo".

Quise terminar con este cuento porque expresa el sentir de una inteligente niña de 5 años. La inmovilidad como sinónimo de muerte, el imperceptible guiño que le hace pensar sin embargo que hay vida interior que está funcionando y que está dirigido exclusivamente a ella; la falta de temor, el recuerdo y por fin el desaparecimiento de ese mágico momento al llegar a la adolescencia.

Un resumen perfecto de cómo entienden la muerte los niños pequeños.

\section{Conflicto de intereses}

La autora declara no tener conflicto de intereses. 


\section{Referencias}

1. Kreicbergs $U$, Valdimarsdóttir $U$, Onelóv E Henter J, Steineck G. Talking about death with children who have severe malignant disease. $\mathrm{N}$ England J M. 2004;351:1175-86.

2. Iacoboni M. Facing yourself. Baby mirrors. In Mirroring people. Eds Farrar, Strauss and Giroux . N York 2008.pags 130-68.

3. Labrell F, Stefianak N. The development of diachronic thinking between 6 and 11 years: The case of growth and death. International J Behavioural Development 2011;35:532-41.

4. Anthony S. Death and time. Inexorable law. In: The discovery of death in childhood and after. Eds. Penguin Education 1973 Great Britain pags 168-205.

5. Piaget J. The origins of intelligence in children Eds WW Norton New York, 1963.

6. Lavados J. Neuropsicología de los principios morales . Boletín Academia Chilena de Medicina 2015;52:59-90.

7. Astuti R. Are we all natural dualists? A cognitive natural approach. J Anthropol Inst 2011;7:429-47.

8. Hopkins M.The development of children's understanding of death. Thesis Doctorate in Clinical Psychology. University of East Anglia 2014

9. Gaab E, Owen G. Caregivers estimations of their children perceptions of death as a biological concept Death Studies
2013;37:693-703.

10. Roa A. Presenta Los cuentos de Charles Perrault. Un estudio antropológico. Editorial Universitaria 2a Ed Santiago Chile. 1988 . pags 11-36.

11. Bonoti F, Leondari A, Mastora A. Exploring children's understanding of death: Through drawings and the death concept questionnaire Death Studies 2013;37:47-60.

12. Lee S, Kim E, Choi Y, Koo J. Cultural variances in composition of biological and supernatural concepts of death: A content analysis of children`s literature . Death Studies 2014;38:538-45.

13. Munro A. Cuento: El ojo. En : Mi vida querida 2013. Random House Mondadori Barcelona . pags 76-81. 